\title{
Research on Different Illumination Image Classification Method
}

\author{
Wenli Zhang ${ }^{1, a}$, Honglu Li, ${ }^{2, b}$, Zhuozheng Wang ${ }^{1, c}$ \\ ${ }^{1}$ The Pilot College of Beijing, Beijing University of Technology, Beijing, China; \\ ${ }^{2}$ The college of electronic information and control engineering, Beijing University of Technology, \\ Beijing, China; \\ aEmail:zhangwenli@bjut.edu.cn, ${ }^{\mathrm{b}}$ Email:lihonglu@emails.bjut.edu.cn, \\ Email:wangzhuozheng@bjut.edu.cn
}

\begin{abstract}
Keywords: different illumination image; feature extraction; support vector machine; BP neural network; K-means
\end{abstract}

\begin{abstract}
Aiming at the problem of video image captured by the monitoring system under the conditions of haze, rain, snow and uneven illumination, a classification method of different illumination is proposed in this paper. Through analyzing the characteristics of different illumination images, the features of different illumination images can be extracted. The different illumination image features can be used to train and construct the classifier. Finally, the different illumination images are classified by the classifier. The experimental results show that the support vector machine (SVM) algorithm, BP neural network algorithm and k-means algorithm all can achieve the classification of different illumination images, and SVM algorithm has the highest classification accuracy and shortest running time.
\end{abstract}

\section{Introduction}

With the development of digital technology, digital monitoring system has been widely used in public places and become an indispensable part of public safety. However, in the face of different weather and light changes in the day, the video obtained under the condition of the haze day or night by the digital monitoring system would be reduced in contrast and dark in quality, which brings difficulties to the subsequent target recognition and tracking. So the video need to be enhanced or restored. But different illumination image processing algorithms are different in the illumination of the monitoring video. In view of the uninterrupted time and unchangeable illumination, it is necessary to manually distinguish different illumination images before illumination process and then choose different algorithms or set different parameters to deal with different illumination image, which exacerbates the difficulty of monitoring the video illumination processing, resulting in complex work, low efficiency and so on. Therefore, there is an urgent need for an automatic classification method to achieve different illumination images to improve this situation. And it is very important to realize automatic classification of different illumination images such as haze, sunny day and night.

In recent years, the domestic and foreign scholars have a lot of different research on image classification. In the following, we introduce the current research of image classification from two aspects: image classification object and image classification method.

(1) According to the different classification objects, it can be classified into classification based on image content, classification based on image scene and classification of remote sensing image, etc. Classification based on image content is to achieve the aircraft, cameras, birds and other content of different categories of images to be classified [1]. Classification based on the image scene is to classify which is the outdoor image or indoor image [2]. Classification based on remote sensing image is to achieve the classification of objects in the hyper spectral remote sensing image [3]. Paper [4] which based on the whole or local features has classified the haze image and the sunny image. But for the monitoring video images, the image which has uneven or lower illumination is more need to classify. If the haze image is the only one that can be classified, it cannot meet the monitoring image following jobs, such as target recognition, tracking, etc. 
(2) According to domestic and foreign scholars, the main methods of image classification are support vector machine(SVM) [5], K-means [6], neural network [7], Bayesian model [8], the method based on word bag model [9] and the improvement and optimization of the above methods [10][2]. For example, [11] used fuzzy support vector machine to classify six kinds of natural images, such as architecture, scenery and animals. In [9], the traditional bag model was improved, which extracted the interest area of image. Then using SVM classifier classified the images of cups, mobile phones, etc. [12] and [13] used the convolution neural network to achieve the classification of natural images, and improved the accuracy of image classification. The above classification method can achieve the image classification, but for different illumination images, the object of classification is a whole or local characteristic, the above method cannot be used for them. Therefore, this paper presents a classification method for different illumination images, aiming at the problems which exist in the research at home and abroad.

Through the analysis of different illumination images, the intensity of image edge, image average and image contrast of different illumination images are extracted as the standard of image classification. Then, the machine learning algorithm is used to train and study the feature data of different illumination images, so as to realize the classification of the image to be measured.

The structure of this paper is as follows: The introduction part introduces the application background of different illumination images and the research status of image classification at home and abroad. The first chapter introduces the principle and method of feature extraction for different illumination images. The second chapter describes the construction of image classifier. The third chapter is the experimental process and experimental results for different illumination images. The fourth chapter summarizes the advantages and disadvantages of this paper, and prospects for future work.

\section{Feature Extraction of Different Illumination Image}

Image feature extraction is the basis of image classification. The image classification is realized by different image features [14]. Through analyzing the different illumination images, which includes haze day image or night image and sunny day image or night image, we found that some features of different illumination images are not the same. A large number of experiments show that image edge intensity, image average and image contrast are the most affected by illumination. Therefore, we regard these three features as the classification criteria for different illumination images. The following describes their principles and calculation methods.

\subsection{The Intensity of Image Edge}

The edge of image [15] reflects the degree of image clarity from the image details. There is a difference in the intensity of image edge of different illumination images in same scene. Therefore, edge intensity values of image can be used as criteria to distinguish different illumination images.

There are a variety of image edge intensity calculation methods [16]. In this paper, we use the sobel operator and the experimental sample of gray image to convolution operation and gain the image intensity of edge detection of the horizontal $G_{x}$ and the vertical $G_{y}$. Then we can calculate the size of the pixel gray with $G_{x}$ and $G_{y}$ as formula (1).

$$
G=\sqrt{G_{x}^{2}+G_{y}^{2}}
$$

According to each edge pixel gray value obtained above, we calculate the average pixel gray edge features of the image, that is, the edge intensity values of the image.

\subsection{The Contrast of Image}

The contrast of image is the degree of light and dark of image contrast [15]. So, different illumination images have different image contrast values. And the contrast of image can tell from different illumination types of image. Image contrast value can be calculated as follows:

$$
c=\sum_{\delta} \delta(i, j) P_{\delta}(i, j)
$$


(2)

Where $i$ is an abscissa pixel and $j$ is an ordinate pixel, $\delta(i, j)=|i-j|$ is the gradation difference between pixels. $P_{\delta}(i, j)$ is the distribution probability of gradation difference between adjacent pixels of $\delta$.

\subsection{The Average of Image}

The average of image is an average of all pixels of the gray image, which is the most basic feature of image [15]. Different images have different gray mean. Therefore, different illumination image can be judged by the average of image. The formula is as follow:

$$
a=\frac{\sum_{\delta} \delta(i, j)}{w \times h}
$$

Where $\delta(i, j)$ represents the pixel of gray image, and $i$ is abscissa pixels and $j$ is ordinate pixels. $w$ and $h$ are the width and height of the image matrix.

According to principle of the above three image features, we calculate the features of different illumination image as shown in Tab.1. From the table, we can see that the features of different illumination images are quite different. Especially the image contrast, the minimum difference of four kinds of different illumination image contrast value is about 40 . So, the image edge intensity, the image contrast and the image average can be used as criterion to tell different illumination image.

Tab.1. The calculate result of different features of different illumination image

\begin{tabular}{|c|c|c|c|c|}
\hline $\begin{array}{c}\text { Type of } \\
\text { image }\end{array}$ & $\begin{array}{c}\text { Sunny day } \\
\text { image }\end{array}$ & $\begin{array}{c}\text { Sunny night } \\
\text { image }\end{array}$ & $\begin{array}{c}\text { Fog and haze } \\
\text { day image }\end{array}$ & $\begin{array}{c}\text { Fog and haze } \\
\text { night image }\end{array}$ \\
\hline $\begin{array}{c}\text { Image edge } \\
\text { intensity }\end{array}$ & $61.0331 \pm 3$ & $25.1659 \pm 3$ & $43.4074 \pm 3$ & $18.4986 \pm 3$ \\
\hline $\begin{array}{c}\text { Image } \\
\text { contrast }\end{array}$ & $223.135 \pm 5$ & $76.6699 \pm 5$ & $116.418 \pm 5$ & $38.5412 \pm 5$ \\
\hline $\begin{array}{c}\text { Image } \\
\text { average }\end{array}$ & $112.796 \pm 3$ & $75.7796 \pm 3$ & $122.601 \pm 3$ & $85.0203 \pm 3$ \\
\hline
\end{tabular}

\section{Achievements of Different Illumination Image Classification}

Machine learning algorithms are commonly used in the field of classification. Different machine learning algorithms have different classification results, different classification accuracy. In this paper, we use three algorithms to construct image classifier achieving different illumination image classification, including support vector machine (SVM) algorithm, back propagation (BP) neural network algorithm and k-means clustering. The following is the construct classifier methods of three algorithms.

\subsection{SVM Classifier Construction}

SVM is a classic two-class classification model. Multi-class classification can be achieved by one-versus-rest method or one-versus-one method based on two-class classification model [17]. In this paper, we use one-versus-rest method to construct the SVM classifier and achieve multi-class classification of different illumination image.

According to the acquired features of images, SVM algorithm based on radial basis kernel function $(\mathrm{RBF})$ is introduced into training. The formula of RBF is as follows.

$$
K(x, z)=\exp \left(-\frac{\|x-z\|^{2}}{2 \sigma^{2}}\right)
$$

Wherein, $x$ is the input feature vector, i.e., the image feature data, wherein $z$ is the center of the kernel function, $\sigma$ is the width of the kernel function. And then, the classification training model is as follows: 


$$
f(x)=\sum_{j=1}^{n} \alpha_{j} y_{j} K\left(x_{j}, z\right)+b
$$

Wherein, $x_{j}$ is the input of image features, i.e., image contrast or edge intensity of image or the average of image, $\alpha_{\mathrm{j}}$ is the Lagrangian, $0<\alpha_{j}<c, c$ is penalty coefficient of wrongly classified sample, $n$ is the number of training samples, $b$ is classification threshold; $y_{i}$ is classification mark, and will be marked -1 or 1 .

We optimize penalty coefficient and RBF kernel width of the above training models through cross-validation method and use the optimal punishment coefficient as the optimal Lagrangian, whereby the SVM classification prediction model is as follows.

$$
f(x)=\sum_{j=1}^{n} \alpha^{*} y_{j} K\left(x_{j}, z\right)+b
$$

Equation (6) is the prediction model of two-class classification based on RBF kernel function of SVM algorithm. Using one-versus-rest method to construct multiple classifiers achieves multi-class classification of different illumination image. We can obtain a plurality of classification prediction models, namely a plurality of image classifiers configured.

\subsection{BP Neural Network Classifier Construction}

BP neural network is a multi-layer feed forward neural network trained by error back propagation algorithm. Its model consists of input layer, hidden layer and output layer [18].

If there are $n$ neurons nodes of input layer, $\left(x_{1}, x_{2}, \ldots, x_{n}\right), p$ neurons nodes of hidden layer, $\left(h_{1}, h_{2}, \ldots, h_{p}\right)$, and $q$ neurons nodes of output layer, $\left(h_{1}, h_{2}, \ldots, h_{q}\right)$. The expected output is $\left(o_{1}, o_{2}, \ldots, o_{q}\right)$. The weight between input layer and hidden layer is $w_{i h}$, the threshold is $\theta_{j}$. The weight between hidden layer and output layer is $w_{h o}$, the threshold is $\theta_{o}$. The actual output of the output node $y_{o}$, the output of hidden node $h_{j}$ and the error of the output node $\varepsilon$ are as follows:

$$
\begin{aligned}
& y_{o}=f\left(\sum w_{h o} h_{j}-\theta_{o}\right) \\
& h_{j}=f\left(\sum w_{i h} x_{i}-\theta_{j}\right) \\
& \varepsilon=\frac{1}{2} \sum_{o=1}^{q}\left(o_{o}-y_{o}\right)^{2}
\end{aligned}
$$

The weights of BP neural network are adjusted by back propagation algorithm, and mean square error and gradient descent method are used to modify the weight of network connection. The training error of the output layer $\delta_{h o}$ and the training error of hidden layer are as follows,

$$
\begin{aligned}
& \delta_{h o}=y_{o}\left(1-y_{o}\right)\left(o_{o}-y_{o}\right) \\
& \delta_{i h}=\sum_{o=1}^{q} \delta_{h o} \times w_{h o}\left[1-x_{j}^{2}\right]
\end{aligned}
$$

The weights and the thresholds of the layers are adjusted by the above-mentioned error training. In the actual network training process, given an input sample and a target output, the iterations are repeated for each training sample. That is, constantly calculating errors, adjusting weights and thresholds until all samples are trained and the error meets the accuracy requirements.

\subsection{K-means Clustering}

K-means algorithm is an iteration algorithm based on distance [19]. It's different from SVM algorithm and BP neural network algorithm which are known as the supervised learning, k-means algorithm is an unsupervised learning and does not need to be supervised learning using the training data. The idea of K-means is cluster the similar data. For the different illumination image, the process is that clustering features data of existed images. The principle of k-means algorithm is as follows.

We assume that the original data set is $\left\{x^{(1)}, x^{(2)}, \ldots, x^{(m)}\right\}$.Under the condition of a given set of classification groups; we select $k$ clustering centers $\mu_{1}, \mu_{2}, \ldots, \mu_{k} \in R^{n}$, then we calculate every data $i$ which center belongs to as follows. 


$$
c^{(i)}:=\arg \min _{j}\left\|x^{(i)}-\mu_{j}\right\|^{2}
$$

For each cluster $\mathrm{j}$, we recalculate the center:

$$
\mu_{j}:=\frac{\sum_{i=1}^{m} 1\left\{c^{(i)}=j\right\} x^{(i)}}{\sum_{i=1}^{m} 1\left\{c^{(i)}=j\right\}}
$$

Repeat the above calculation until each cluster center converges, we can acquire the final clustering results. Through clustering the extracted features data of different illumination images, we can achieve different illumination image classification.

\subsection{Image Classification Algorithm}

According to SVM algorithm, BP neural network algorithm and K-means algorithm to construct the image classifier, the experimental algorithm steps of the classification of different illumination images are as follows:

Step 1: According to the selected experimental images of different illumination, the feature of image edge intensity, image contrast feature and image average feature were extracted respectively, saving the characteristic data and determining the data training set and test set;

Step 2: The training set of the image feature data is trained and clustered by SVM, BP neural network and $\mathrm{k}$-means algorithm respectively, constructing the image classifier.

Step 3: Importing the test set data into the trained classifier, we will test the accuracy of the test set according the constructed image classifier.

\section{Experimental Results}

\subsection{Implementation}

In this paper, road monitoring images at the same place and at different time periods are used as experimental data. We have collected four kinds of different illumination images in monitoring system, including sunny day image, sunny night image, fog and haze image and fog and haze night image. Every kind of video is taken for thirty minutes; the daytime video is taken in 10:00am 11:00am and the evening video is taken in 20:00pm 21:00pm. The taken videos of images are shown as Fig.3.1. In the collected surveillance video, 300 frames are taken as the experimental image every ten minutes from the start of the video. So there are 1200 experimental images for each illumination type. Each image is $640 \times 352$ pixels. In experiment, 800 images are randomly selected as training samples, and the remaining 400 samples are selected as test samples. MATLAB2012a is used.

We extract the features from experimental images in experiment firstly, namely the image edge intensity, image contrast feature and image average, and then use these features to train or cluster on the basis of three algorithms of SVM, BP natural network and k-means respectively. Finally, we achieve the classification of the experimental test set using the classifiers which have been trained or clustered.
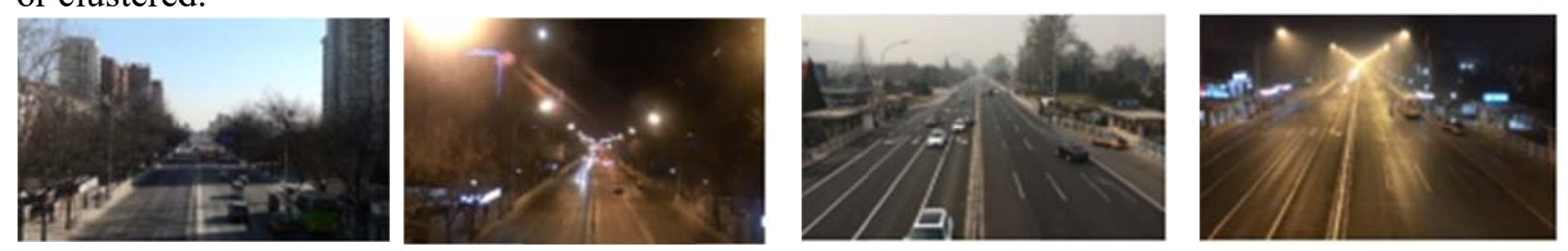

a.sunny day image

b.sunny night image c.fog and haze day image

d.fog and haze night image

Fig.1. The collected images of different illumination

\subsection{Experiment Results and Analysis}

We experimented with four kinds of different illumination images. SVM classifier, BP neural 
network classifier and k-means algorithm are used to classify the different illumination images. We compare the classification accuracy and algorithm running time of the three classification algorithms and analyze the advantages and disadvantages of each classifier. The results are shown in the following tables.

Tab.2. Classification results of different illumination images with image edge intensity

\begin{tabular}{|c|c|c|}
\hline Type of algorithm & $\begin{array}{c}\text { Classification } \\
\text { accuracy }\end{array}$ & Running time \\
\hline SVM algorithm & $86.25 \%$ & $2.63 \mathrm{~s}$ \\
\hline $\begin{array}{c}\text { BP neural network } \\
\text { algorithm }\end{array}$ & $78.03 \%$ & $184 \mathrm{~s}$ \\
\hline $\begin{array}{c}\text { K-means } \\
\text { algorithm }\end{array}$ & $77.23 \%$ & $1.52 \mathrm{~s}$ \\
\hline
\end{tabular}

Tab.3. Classification results of different illumination images with image contrast

\begin{tabular}{|c|c|c|}
\hline Type of algorithm & $\begin{array}{c}\text { Classification } \\
\text { accuracy }\end{array}$ & Running time \\
\hline SVM algorithm & $85.71 \%$ & $2.59 \mathrm{~s}$ \\
\hline $\begin{array}{c}\text { BP neural network } \\
\text { algorithm }\end{array}$ & $82.56 \%$ & $152 \mathrm{~s}$ \\
\hline $\begin{array}{c}\text { K-means } \\
\text { algorithm }\end{array}$ & $90.12 \%$ & $1.39 \mathrm{~s}$ \\
\hline
\end{tabular}

Tab.4. Classification results of different illumination images with image average

\begin{tabular}{|c|c|c|}
\hline Type of algorithm & $\begin{array}{c}\text { Classification } \\
\text { accuracy }\end{array}$ & Running time \\
\hline SVM algorithm & $88.95 \%$ & $2.18 \mathrm{~s}$ \\
\hline $\begin{array}{c}\text { BP neural network } \\
\text { algorithm }\end{array}$ & $83.46 \%$ & $103 \mathrm{~s}$ \\
\hline $\begin{array}{c}\text { K-means } \\
\text { algorithm }\end{array}$ & $80.32 \%$ & $1.98 \mathrm{~s}$ \\
\hline
\end{tabular}

From the above experimental results, we can see that different image features have different classification accuracy. To compare three kinds of image classification results, the average classification precision of image contrast is the highest, which is $86.13 \%$. The result is related to the large difference contrast value of different illumination image. The larger difference of features between different categories is, the higher the classification accuracy is. To compare three kinds of image classification algorithms, SVM algorithm has the highest average classification accuracy, followed by BP neural network algorithm. K-means algorithm has higher classification accuracy randomly. K-means algorithm does not need to be trained, which determines the classification results by calculating the distance between features of data. If the distance between features of data is small, it is prone to make a mistake of classification, so that the classification accuracy of probability is random.

The results of running time of the three algorithms in tables show that SVM algorithm running time is slightly higher than $\mathrm{K}$-means algorithm, BP neural network algorithm takes a long time. And BP neural network algorithm in the process of training is easy to fall into the local minimum point, affecting the final classification accuracy. So it is difficult to real-time applications. In summary, SVM algorithm is the best choice to achieve different illumination image classification among three algorithms of image classification in this paper. 


\section{Conclusions}

In this paper, we focus on the problem of multiple illumination images in the surveillance video. By extracting the edge intensity feature, contrast feature and image average feature of the image and using the features to construct the image classifiers through machine learning algorithm and clustering algorithm, we have achieved the classification of different illumination images. In the experiment, we compare the classification accuracy and running time of SVM algorithm, BP neural network algorithm and K-means algorithm. The results show that image contrast is the best criteria for distinguishing different illumination images. In terms of image classification algorithm, BP neural network algorithm and K-means algorithm can achieve different illumination image classification, but the running time of BP neural network algorithm is longer than the other and $\mathrm{K}$-means algorithm is too dependent on the image feature data. These shortcomings make BP neural network algorithm and K-means algorithm not be applied in the monitoring system. Therefore, SVM algorithm can not only ensure the accuracy of classification, but also meet the time requirement of image classification to realize the classification of different illumination images in monitoring video.

Whether in the selection of image features or the choice of classification methods, this paper is to explore the classification of different illumination images in surveillance video. We will research and optimize the SVM algorithm to improve the classification accuracy while shortening the running time of the algorithm, and further improve the algorithm performance of different illumination image classification.

\section{Acknowledgment}

The research has been sponsored by the foundation of the Ministry of Education of China for returned scholars (Project No. 20141685) and the basic research foundation of Beijing University of Technology for research on high performance image fusion (Project No. 023000514315501).

\section{References}

[1] YANG Jie, CHEN Xiaoyun, Comparison of Approaches for Image Classification[J], Microcomputer Applications, 2007,28(6)

[2] YUAN Lu, Feature Extraction Algorithm Optimization and Implementation Used for The Scene Image Classification[D], Beijing University of Posts and Telecommunications, 2012

[3] WANG Yida, SHEN Xiling, XIE Jong, A Review of Remote Sensing Image Classification Method[J], Remote sensing information, 2006

[4] YU Xiaoliang, The Research of Fog Image Discrimination and Dehazing(D), Beijing University of Technology, 2012

[5] ZHANG Shuya, ZHAO Yiming, LI Junli, Algorithm and implementation of image classification based on SVM[J], Computer Engineering and Applications 2007,43(25)

[6] JU Hongyun, ZHANG Junben, LI Chaofeng, WANG Zhengyou, Automated remote sensing image classification method based on K-means and SVM[J], Application Research of Computers,2007,24(11)

[7] XIE Wenlan, SHI Yuexiang, XIAO Ping, Classification of natural image based on BP neural network[J], Computer Engineering and Applications,2010,46(2)

[8] CHENG Huanhuan, Image Content Representation and Classification Based on Bayesian Networks[D], National university of defense technology, 2011

[9] ZHAO Chunhui, WANG Ying, Masahide KANEKO, An Optimized Method for Image Classification Based on Bag of Words Model[J], Journal of Electronics \& Information Technology, 2012,34(9) 
[10]WANG Xiaoyun, Dissertation Submitted to Shanghai Jiao Tong University for the Degree of Master[D], Shanghai jiaotong university, 2012

[11]CAO Jianfang, JIAO Lijuan, Image Classification Algorithm Based on Fuzzy Support Vector Machine[J], Computer \& Digital Engineering,2013(4)

[12]XIE Baojian, The Research of Image Classification Method Based on Convolution Neural Network(D), Hefei University of Technology, 2015

[13]CHUN Minnan, Research of Image Classification Technology Based On Convolutional Neural Network(D), Xiangtan University, 2015

[14]LIU li, KUANG Gangyao, Overview of Image Textural Feature Extraction Methods[J], Journal of Image and Graphics, 2009,14(4)

[15]Rafael C. Gonzalez, Richard E. Woods, Steven L.Eddins. Translator: RUAN Qiuqi. Digital Image Processing (Second edition), Beijing: Publishing House of Electronics Industry, 2007.8

[16]DUAN Ruiling, LI Qingxiang, LI Yuhe, Summary of image edge detection[J], Optical Technique, 2005,31(3)

[17]HUANG Yong, ZHENG Chunying, SONG Zhonghu, Multi-class Support Vector Machines algorithm summarization[J], Computing Technology and Automation, 2005, 24(4)

[18]SUN JunDing,LI Lin, Classification of Medical Image Based on BP Neural Network[J], Computer Systems Application,2012(21)

[19] Wu Suhui, Cheng Ying, Zheng Yanning, Pan Yuntao, Survey on K-means Algorithm[J], Modern Library and Information Technology, 2011,205(5) 